\title{
Interaction of Salmonella serotypes with porcine macrophages in vitro does not correlate with virulence
}

\author{
Patricia R. Watson, Sue M. Paulin, Philip W. Jones and Timothy S. Wallis
}

Author for correspondence: Timothy S. Wallis. Tel: +44 1635 577230. Fax: +44 1635577263.

e-mail: timothy.wallis@bbsrc.ac.uk

Institute for Animal Health, Compton, Newbury RG20 7NN, UK
The interaction between Salmonella serotypes and macrophages is potentially instrumental in determining the outcome of infection. The nature of this interaction was characterized with respect to virulence and serotype-host specificity using pigs as the infection model. Experimental infection with Salmonella typhimurium, Salmonella choleraesuis or Salmonella dublin resulted in enteric, systemic or asymptomatic infection, respectively, which correlates well with the association of $S$. choleraesuis with systemic disease in pigs in epidemiological studies. Persistence within porcine alveolar macrophages in vitro did not directly correlate with virulence since $S$. typhimurium persisted in the highest numbers, and $S$. choleraesuis in the lowest. Comparison to other studies revealed that the relatively high persistence of $S$. typhimurium in macrophages correlates with its virulence in a broad range of animals: this could be a virulence mechanism for broad-hostrange serotypes. There were little or no significant differences in the induction of pro-inflammatory cytokines by macrophages infected with the three serotypes. S. typhimurium and $S$. dublin, but not $S$. choleraesuis, damaged porcine macrophages, and the mechanism of damage did not resemble apoptosis. In conclusion, the virulence of Salmonella serotypes in pigs did not directly correlate with their interaction with porcine macrophages in vitro. The interaction of Salmonella and macrophages in vitro may not accurately model their interaction in vivo, and this will form the basis of further study.

Keywords: Salmonella serotypes, porcine virulence, macrophages, apoptosis, cytokine bioassays

\section{INTRODUCTION}

Salmonellosis is an important zoonosis ranging in severity from subclinical infection, through mild diarrhoea, to severe systemic disease. One of its characteristics is that some serotypes, for example Salmonella typhimurium, have a broad host range, whereas several other serotypes, for example, Salmonella choleraesuis, Salmonella typhi, Salmonella dublin and Salmonella pullorum, are associated with disease in specific, and usually a very limited, range of host animals (pigs, humans, cattle and poultry, respectively). This has been termed serotype-host specificity, host adaptation or host restriction, although for the sake of clarity, only the first of these terms will be used here. In pigs, Salmonella

Abbreviation: LDH, lactate dehydrogenase. infections are associated with significant animal suffering and economic losses and pigs may be a reservoir of infection for humans (Berends et al., 1997). Natural infection of pigs with $S$. choleraesuis is usually associated with systemic disease, whereas infection with $S$. typhimurium is associated with enteric disease (reviewed by Wilcock \& Schwartz, 1992). This is analogous to $S$. typhi and S. typhimurium infections in humans. It is possible to infect pigs experimentally with $S$. choleraesuis and S. typhimurium, and the resulting disease is similar to that reported in the field (Reed et al., 1986). Therefore pigs are a good model for studying the pathogenesis of systemic and enteric forms of human salmonellosis and for investigating the mechanisms of serotype-host specificity.

The factors influencing Salmonella serotype hostspecificity remain poorly defined, although there is some 
evidence that bacterial survival within macrophages is important. S. typhimurium can persist in significantly higher numbers than $S$. typhi in primary murine macrophages in vitro (Lissner et al., 1985; Vladoianu et al., 1990; Alpuche-Aranda et al., 1995; Ishibashi \& Arai, 1996; Schwan \& Kopecko, 1997), which correlates with the virulence of these serotypes for mice. Similarly, S. typhi persists better in human compared to murine macrophages in vitro, which again correlates with virulence. However, the relative persistence of $S$. typhi and $S$. typhimurium in human macrophages varies considerably between different studies (Vladoianu et al., 1990; Alpuche-Aranda et al., 1995; Ishibashi \& Arai, 1996). This variation may be explained by unquantified differences in the magnitude of macrophage lysis, which can have a major effect on the interpretation of results from bacterial persistence studies (Guilloteau et al., 1996; Sizemore et al., 1997).

This study has used Salmonella serotypes of defined virulence in pigs to characterize the interaction of Salmonella serotypes with porcine macrophages. Bacterial persistence, the magnitude and nature of Salmonella-induced macrophage damage, and the production of pro-inflammatory cytokines were assessed and correlated with virulence.

\section{METHODS}

Bacterial strains. S. typhimurium strains ST4/74 and ST12/75, S. dublin strains SD2229 and SD3246 and S. choleraesuis var. kunzendorf strains SCSA50 and SCS14/74 have been described previously and extensively characterized (Baird et al., 1985; Wallis et al., 1995; Guilloteau et al., 1996; Watson et al., 1998; Bolton et al., 1999). To date, there is no evidence that there are any differences between the two strains of each serotype in a variety of in vitro and in vivo assays. Strains were stored as cultures in Luria-Bertani broth containing 30\% glycerol at $70{ }^{\circ} \mathrm{C}$ and were streaked onto MacConkey plates and incubated at $37^{\circ} \mathrm{C}$ overnight for use. Inocula for all of the in vitro assays were prepared by inoculating $10 \mathrm{ml}$ LuriaBertani broth with several freshly streaked colonies and incubating overnight at $37^{\circ} \mathrm{C}$ with shaking at 150 r.p.m. The cultures were subcultured 1 in 100 into fresh broth and incubated as before for $4 \mathrm{~h}$. The number of bacteria present in each subculture was estimated by spectrophotometry, the subcultures were diluted to the required number of c.f.u. $\mathrm{ml}^{-1}$, and the bacterial concentration was determined by viable count. Heat-inactivated bacteria were prepared by placing the diluted subcultures in a boiling water bath for $10 \mathrm{~min}$.

Virulence of Salmonella serotypes following inoculation of pigs. Camborough pigs (6-7 weeks old) were supplied by the Institute for Animal Health farm. Pigs were weaned at 2 weeks and given a $3 \mathrm{~d}$ course of enrofloxacin (Baytril; Bayer), to compensate for their lowered resistance to bacterial infections during weaning and transport to the disease-secure animal unit. Thereafter, pigs were fed on a diet of antibioticfree, irradiated pellets. None of the pigs excreted salmonellas at 0,2 or 4 weeks before infection as determined by enrichment of faecal swabs in Rappaport broth (at $37^{\circ} \mathrm{C}$ for $18 \mathrm{~h}$ ) and selenite brilliant green broth (at $42^{\circ} \mathrm{C}$ for $18 \mathrm{~h}$ ) followed by overnight incubation at $37^{\circ} \mathrm{C}$ on modified brilliant green agar (Difco).

Bacterial cultures of S. typhimurium ST4/74, S. dublin SD3246 or S. choleraesuis SCS14/74 were prepared by inoculating Bacto-Tryptose broth (Jones, 1975) with several bacterial colonies and incubating statically at $37^{\circ} \mathrm{C}$ for $18 \mathrm{~h}$. For oral inoculation, approximately $3 \times 10^{8}$ c.f.u. were suspended in $10 \mathrm{ml}$ ant-acid $\left[5 \% \mathrm{Mg}\left(\mathrm{SiO}_{3}\right)_{3}, 5 \% \mathrm{NaHCO}_{3}\right.$ and $5 \% \mathrm{MgCO}_{3}$ in $\mathrm{H}_{2} \mathrm{O}$ ] and administered directly into the stomach of each pig immediately before the morning feed using a $10_{\mathrm{FG}}$ catheter (Arnolds Veterinary Products). For intravenous inoculation, approximately $1 \times 10^{6}$ c.f.u. in a volume of $1 \mathrm{ml}$ were injected into the superior vena cava. Rectal temperatures were recorded and animals were monitored for clinical signs of disease twice daily. All animals were humanely killed at $7 \mathrm{~d}$ after inoculation. The numbers of bacteria in the lung, spleen, liver, ileum, caecum and colon and in the lymph nodes associated with these sites were determined by viable counts of one sample per site as described by Watson et al. (1998). The limit of accurate quantification was $2 \cdot 0 \log _{10}$ c.f.u. (g tissue $)^{-1}$ and any samples containing fewer bacteria were enriched in Rappaport and selenite brilliant green broth as described above. Samples that were positive on enrichment were given a value of $2 \cdot 0 \log _{10}$ c.f.u. $\mathrm{g}^{-1}$ and those that were negative were given a value of 0 c.f.u. $\mathrm{g}^{-1}$.

Salmonella infection of porcine alveolar macrophages and preparation of conditioned supernatants for cytokine bioassay. Alveolar macrophages were isolated from healthy Camborough pigs by bronchoalveolar lavage as described previously for cattle (Guilloteau et al., 1996). Viability of cells, of which $90-99 \%$ are alveolar macrophages (Ganter \& Hensel, 1997), was $>99 \%$ as assessed by trypan blue exclusion. Cells were allowed to adhere to 24-well tissue culture plates at $5 \times 10^{5}$ cells $\mathrm{ml}^{-1}$ in Iscove's modified Dulbecco's medium containing $10 \%$ foetal calf serum (FCS), 100 units penicillin $\mathrm{ml}^{-1}, 100 \mu \mathrm{g}$ streptomycin $\mathrm{ml}^{-1}$ and $100 \mu \mathrm{g}$ gentamicin $\mathrm{ml}^{-1}$ and incubated overnight at $37^{\circ} \mathrm{C}$ in $5 \% \mathrm{CO}_{2}$ and $95 \%$ humidity. Two hours before infection, non-adherent cells were washed off and the culture medium was changed to Dulbecco's modified Eagle's medium Ham's nutrient mix F-12 without phenol red (DME/F12) containing $5 \%$ FCS. Bacterial cultures were prepared as described above and diluted in DME/F12 medium to give a ratio of infection of five bacteria per macrophage. Viable and heat-inactivated bacteria were added to the macrophage monolayers and the monolayers were incubated for $1,3,24$ or $48 \mathrm{~h}$. Overgrowth of extracellular bacteria in monolayers incubated for 24 or $48 \mathrm{~h}$ was prevented by washing the monolayers $1 \mathrm{~h}$ after infection and adding DME/F12 containing 5\% FCS and $100 \mu$ gentamicin $\mathrm{ml}^{-1}$, followed by washing the monolayers after a further hour and adding DME/F12 containing $5 \%$ FCS and $10 \mu \mathrm{g}$ gentamicin $\mathrm{ml}^{-1}$. Gentamicin was not added to the monolayers incubated for 1 or $3 \mathrm{~h}$, because changing the media would have prevented the measurement of cytokines released immediately after infection. After the appropriate incubation time, the supernatants, termed conditioned macrophage supernatants, were collected and centrifuged $\left(13500 \mathrm{~g}, 2 \mathrm{~min}, 4{ }^{\circ} \mathrm{C}\right)$ to reduce the amount of cell debris and bacteria. An aliquot $(50 \mu \mathrm{l})$ was used to quantify macrophage damage by measuring the amount of lactate dehydrogenase $(\mathrm{LDH})$ released by the monolayers using the cytotoxic 96 kit (Promega). Results obtained using this method correlate with the severity of macrophage damage by microscopic assessment (Guilloteau et al., 1996). The results were expressed as the percentage of LDH released by infected monolayers compared to the LDH released by detergent treatment of control monolayers. The remaining conditioned macrophage supernatants were stored at $-70{ }^{\circ} \mathrm{C}$ in aliquots. The number of macrophage-associated bacteria was determined by washing monolayers twice with 
DME/F12 without supplements and adding $1 \mathrm{ml}$ PBS containing $0 \cdot 1 \%$ sodium deoxycholate. Serial dilutions of the lysates were plated onto MacConkey agar plates and incubated overnight at $37^{\circ} \mathrm{C}$.

Bioassay for IL-1 activity. Cytokine release was quantified using biological assays, which have the following characteristics : they measure cytokine release rather than expression; they are very sensitive; and they are unlikely to be affected by unrecognized inhibitors often present in serum, when using monocultures of cells. In addition, ELISAs for porcine cytokines are not readily available. IL-1 activity was measured using the A375 cell line. The growth of A375 cells is inhibited in the presence of human and porcine IL-1 (Nakai et al., 1988; Cendan et al., 1994). The cells were maintained in Eagle's minimal essential medium with Earle's salts containing $10 \%$ FCS, 100 units penicillin $\mathrm{ml}^{-1}, 100 \mu \mathrm{g}$ streptomycin $\mathrm{ml}^{-1}$ and $100 \mu \mathrm{g}$ gentamicin $\mathrm{ml}^{-1}$. Aliquots of the conditioned macrophage supernatants were thawed and serially diluted 1 in 2 in the above media to give a final volume of $50 \mu \mathrm{l}$ per well, in 96well plates. Medium alone was used as a negative control. Fifty microlitres of A375 at $1 \times 10^{5}$ cells ml $\mathrm{ml}^{-1}$ in Eagle's minimal essential medium with supplements was added to each well and incubated for $96 \mathrm{~h}$ at $37^{\circ} \mathrm{C}$ in $5 \% \mathrm{CO}_{2}$ and $95 \%$ humidity. Following incubation, the culture medium was removed and the wells were washed once with PBS. The remaining cells were stained with freshly prepared crystal violet stain $(0.1 \%$ crystal violet, $1 \%$ acetic acid, $5 \%$ formaldehyde, $4 \cdot 25 \mathrm{~g} \mathrm{NaCl} \mathrm{l}^{-1}, 2 \cdot 25 \mathrm{~g} \mathrm{NaH}_{2} \mathrm{PO}_{4}$ anhydrous $\mathrm{l}^{-1}$, $2.75 \mathrm{~g} \mathrm{Na}_{2} \mathrm{HPO}_{4}$ anhydrous $\mathrm{l}^{-1}$ ) for $2 \mathrm{~h}$ at room temperature and then the excess stain was removed by washing with PBS. The retained crystal violet was dissolved in $100 \%$ methanol and quantified by spectrophotometry $\left(\mathrm{OD}_{595}\right)$. The percentage growth inhibition was calculated using the formula: $\left[\left(\mathrm{OD}_{595}\right.\right.$ negative control $-\mathrm{OD}_{595}$ test sample)/OD $\mathrm{OD}_{55}$ negative control] $\times 100$. The range of detection of the assay was $0 \cdot 1$ units per well (resulting in $7 \%$ growth inhibition) to $>10$ units per well (resulting in $75 \%$ growth inhibition) as estimated from a dose response curve to recombinant human IL- $1 \alpha$ (Genzyme Diagnostics) which was determined in parallel with each assay.

Bioassay for IL-6 activity. IL-6 activity was measured using the 7TD1 cell line (kindly supplied by P. Kaiser, IAH, Compton, UK), whose growth is dependent on the presence of IL-6 (Van Snick et al., 1986). The suitability of this cell line in a bioassay for porcine IL-6 has been reported previously (Frank et al., 1996). The cells were maintained in RPMI 1640 containing $10 \%$ FCS, 100 units penicillin $\mathrm{ml}^{-1}, 100 \mu \mathrm{g}$ streptomycin ml $\mathrm{m}^{-1}$, $100 \mu \mathrm{g}$ gentamicin $\mathrm{ml}^{-1}, 0.05 \mathrm{mM} 2$-mercaptoethanol. Recombinant murine IL-6 (R\&D Systems) was added at a concentration of $4 \mathrm{ng} \mathrm{ml}^{-1}$ for routine growth of the cells. For use in the bioassay, cells were washed three times to remove IL-6 and were resuspended in RPMI 1640 with supplements, but without IL-6. Aliquots of the conditioned macrophage supernatants were thawed and serially diluted 1 in 5 in RPMI 1640 with supplements to give a final volume of $50 \mu \mathrm{l}$ per well, in 96well plates. Fifty microlitres of 7TD1 cell suspension at $1 \times 10^{5}$ cells $\mathrm{ml}^{-1}$ in RPMI with supplements was added to each well. The assay plates were incubated for $72 \mathrm{~h}$ at $37^{\circ} \mathrm{C}$ in an atmosphere of $5 \% \mathrm{CO}_{2}$ and $95 \%$ humidity. Growth of the 7TD1 cells was estimated by measuring DNA synthesis. An aliquot of $\left[{ }^{3} \mathrm{H}\right]$ thymidine containing $0.015 \mathrm{MBq}$ radioactivity was added to each well $18 \mathrm{~h}$ before the end of the incubation period. The cells were harvested onto glass fibre filters and incorporation of $\left[{ }^{3} \mathrm{H}\right]$ thymidine into the cells was measured using a betaplate liquid scintillation counter. The range of detection was $0.001 \mathrm{pg} \mathrm{ml}^{-1}$ (5000 c.p.m. incorporated triti- ated thymidine) to greater than $8 \mathrm{pg} \mathrm{ml}^{-1}$ (45000 c.p.m.) as estimated from a dose response curve to murine IL-6 determined in parallel to each assay.

Bioassay for TNF- $\alpha$ activity. TNF- $\alpha$ activity was measured using the WEHI 164 clone 13 cell line (kindly supplied by G. Entrican, Moredun Research Institute, Edinburgh, UK). This cell line is sensitive to the cytotoxic activity of human TNF- $\alpha$ (Espevik \& Nissen-Meyer, 1986) and is suitable as a bioassay for porcine TNF- $\alpha$ (Kim et al., 1991). Cells were maintained in Dulbecco's modified essential medium containing $10 \%$ FCS, 100 units penicillin $\mathrm{ml}^{-1}, 100 \mu \mathrm{g}$ streptomycin $\mathrm{ml}^{-1}$ and $100 \mu \mathrm{g}$ gentamicin $\mathrm{ml}^{-1}$. Semi-confluent monolayers were prepared by adding $3 \times 10^{4}$ WEHI cells into wells of a 96-well plate and incubating overnight at $37{ }^{\circ} \mathrm{C}$ in an atmosphere of $5 \% \mathrm{CO}_{2}$ and $95 \%$ humidity. The medium overlying the monolayers was replaced with $50 \mu \mathrm{l}$ fresh medium. Aliquots of the conditioned macrophage supernatants were thawed and serially diluted 1 in 2 into the medium overlying the monolayers to give a final volume of $50 \mu \mathrm{l}$. Medium alone was used as a negative control. Finally, $50 \mu \mathrm{l}$ media containing $0.5 \mu \mathrm{g}$ actinomycin D-mannitol $\mathrm{ml}^{-1}$ (Sigma) was added to each well. The monolayers were incubated for $18 \mathrm{~h}$ as above. Following incubation, the remaining cells were stained with crystal violet stain as described for the IL-1 bioassay. The percentage cytotoxicity was calculated according to the formula: $\left[\left(\mathrm{OD}_{595}\right.\right.$ negative control $-\mathrm{OD}_{595}$ test sample $) /$ $\mathrm{OD}_{595}$ negative control] $\times 100$. The range of detection was $0 \cdot 001$ units per well ( $3 \%$ cytotoxicity) to greater than $0 \cdot 1$ units per well ( $85 \%$ cytotoxicity), as estimated from a dose response curve to recombinant human TNF- $\alpha$ (Genzyme Diagnostics) determined in parallel to each assay.

Characterization of DNA from macrophages. Porcine alveolar macrophages were isolated and maintained in 24-well plates as described above. Bacterial cultures were prepared as described above and, where required, were opsonized in $10 \%$ autologous serum for $40 \mathrm{~min}$ on a rolling platform. The monolayers were infected and subsequently incubated as described above: the overgrowth of bacteria in the culture medium of infected monolayers incubated for 24 and $48 \mathrm{~h}$ was prevented with the use of gentamicin. Actinomycin D-mannitol was added to three control monolayers to give a final concentration of $1 \mu \mathrm{g} \mathrm{ml}^{-1}$. DNA was extracted from macrophages by the method of Zychlinsky et al. (1992) with minor modifications as follows. Monolayers were washed twice with PBS and $200 \mu \mathrm{l}$ lysis buffer (10 mM Tris; $5 \mathrm{mM}$ EDTA; $0.5 \%$ SDS) was added and incubated at room temperature for $15 \mathrm{~min}$. The lysate was pooled from triplicate wells, $1 \mu \mathrm{l} 20 \mathrm{mg}$ proteinase $\mathrm{K} \mathrm{ml}^{-1}$ was added and the mixture was incubated for $2 \mathrm{~h}$ at $56^{\circ} \mathrm{C}$. An equal volume of Tris-saturated phenol/ chloroform $(1 / 1, \mathrm{v} / \mathrm{v})$ was added and mixed gently for $5 \mathrm{~min}$. The mixture was centrifuged $(13500 \mathrm{~g}$, room temperature, 15 $\min$ ) and the upper aqueous layer was recovered. DNA was precipitated overnight at $-20^{\circ} \mathrm{C}$ by the addition of $1 / 10$ volume $3 \mathrm{M}$ sodium acetate and 2 vols ethanol and collected by centrifugation $(13500 \mathrm{~g}$, room temperature, $20 \mathrm{~min})$. The DNA was washed once in $70 \%$ ethanol, air-dried and dissolved in $30 \mu \mathrm{l}$ TE buffer containing $10 \mu \mathrm{g}$ RNase $\mathrm{ml}^{-1}$. Samples $(10 \mu \mathrm{l})$ were separated through a $1.5 \%$ agarose gel and examined on a UV transilluminator.

Electron microscopy of macrophages. Porcine alveolar macrophages were isolated and maintained (at $30 \mathrm{ml}$ of $1 \times 10^{5}$ cells $\mathrm{ml}^{-1}$ in $250 \mathrm{ml}$ flasks) as described above. Monolayers were infected with bacteria with or without opsonization as described above. Actinomycin D-mannitol was added to control monolayers to give a final concentration of $1 \mu \mathrm{g} \mathrm{ml} l^{-1}$. 
At $24 \mathrm{~h}$ after infection, the monolayers were washed once with $0 \cdot 1 \mathrm{M}$ cacodylate-buffered $2 \cdot 5 \%$ glutaraldehyde and incubated overnight. The fixed macrophages were washed twice in $0 \cdot 1 \mathrm{M}$ phosphate buffer for $30 \mathrm{~min}$ and then post-fixed in $1 \% 0.1 \mathrm{M}$ phosphate-buffered osmium tetroxide for $90 \mathrm{~min}$. The macrophages were washed five times in double-distilled water and resuspended in $1 \%$ aqueous uranyl acetate for $1 \mathrm{~h}$ in the dark. After a further wash in water, the macrophages were embedded in $0.5 \mathrm{ml} 1 \%$ agar. The solidified agar blocks were cut into $2 \mathrm{~mm}^{3}$ cubes, dehydrated through a methanol gradient and incubated for $2 \times 15 \mathrm{~min}$ in propylene oxide. The blocks were embedded in araldite resin and incubated at $60{ }^{\circ} \mathrm{C}$ for $48 \mathrm{~h}$ to allow polymerization to occur. Blocks were sectioned at a thickness of approximately $90 \mathrm{~nm}$ and the sections were collected on 3-mm-diameter nickel Athene grids and stained with lead citrate for $10 \mathrm{~min}$. The sections were examined using a Philips EM 300 transmission electron microscope at an accelerating voltage of $80 \mathrm{kV}$.

\section{RESULTS}

\section{S. typhimurium is moderately virulent, S. dublin is of low virulence and $S$. choleraesuis is highly virulent for pigs}

The relative virulence of $S$. typhimurium, S. dublin and $S$. choleraesuis in pigs was assessed following oral inoculation with approximately $3 \times 10^{8}$ c.f.u. Clinical symptoms were monitored for $7 \mathrm{~d}$ after inoculation and the number of salmonellas present in several intestinal and systemic sites was determined on the seventh day at post-mortem. S. typhimurium induced a rapid, but short-lived, increase in rectal temperature (Fig. 1), and during this time the pigs were diarrhoeic, vomiting and appeared depressed. Two to three days after inoculation, clinical symptoms ceased and temperatures returned to normal. At post-mortem, salmonellas were recovered at between 2.5 and $4 \cdot 0 \log _{10}$ c.f.u. $\mathrm{g}^{-1}$ from all three regions of intestinal wall and associated nodes, but were not recovered from the systemic sites in quantifiable numbers (Fig. 2a). Pigs inoculated with S. dublin had no clinical symptoms and only a mild and short-lived

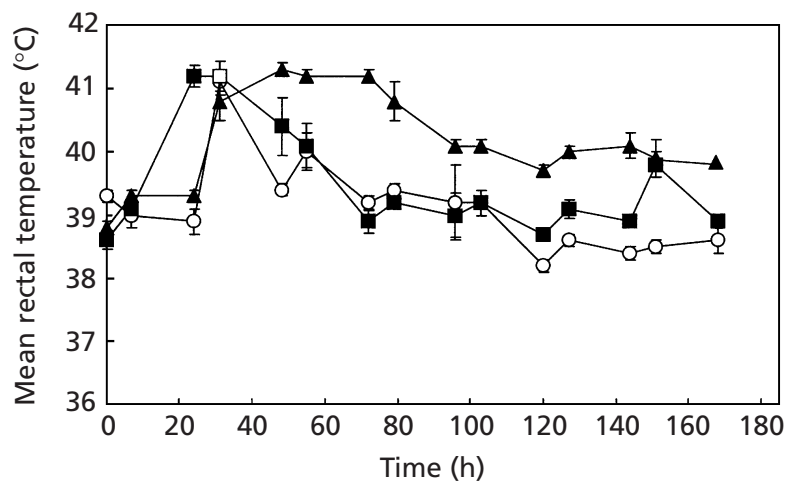

Fig. 1. Rectal temperatures of pigs following oral inoculation with S. typhimurium ( $\boldsymbol{\square})$, S. dublin $(\bigcirc)$ or S. choleraesuis $(\boldsymbol{A})$. Each datum point is the mean of three pigs and is presented with the standard error of the mean. pyrexic response. S. dublin was recovered in relatively low numbers from the intestinal sites (approximately $2 \cdot 0-3 \cdot 0 \log _{10}$ c.f.u. $\mathrm{g}^{-1}$ ), and not at all from the systemic sites. S. choleraesuis induced the most prolonged symptoms of the three serotypes. All the pigs had severe pyrexia, intermittent diarrhoea and appeared depressed and lethargic for the duration of the experiment. $S$. choleraesuis was recovered in significantly higher numbers $(P<0.05)$ than either $S$. typhimurium or $S$. dublin from the intestinal sites and was the only serotype recovered from the systemic sites in quantifiable numbers (approximately $4 \cdot 0 \log _{10}$ c.f.u. $\mathrm{g}^{-1}$ ).

Similar results were obtained following intravenous inoculation of pigs with $1 \times 10^{6}$ c.f.u. S. choleraesuis was the only serotype to induce disease symptoms, with a pyrexic response from day 4 and onwards, and was the only serotype recovered in quantifiable numbers from systemic sites (approximately $5 \cdot 0 \log _{10}$ c.f.u. $\mathrm{g}^{-1}$ ) (Fig. $2 b)$.

\section{S. typhimurium and S. dublin persist within, and induce damage to, porcine alveolar macrophages at significantly greater magnitude than $\mathbf{S}$. choleraesuis}

The relative recovery of S. typhimurium, S. dublin and S. choleraesuis was assessed up to $48 \mathrm{~h}$ after infection of porcine alveolar macrophages. Gentamicin was added after $1 \mathrm{~h}$ of infection to macrophages incubated for 24 or $48 \mathrm{~h}$ to prevent overgrowth of extracellular bacteria. Non-adherent bacteria and gentamicin were removed by washing the macrophages immediately before lysing the macrophages for bacterial recovery. Therefore, the bacterial recovery at 1 and $3 \mathrm{~h}$ is obtained from adherent and intracellular bacteria, and at 24 and $48 \mathrm{~h}$ from only intracellular bacteria. The number of bacteria associated with the macrophages at $1 \mathrm{~h}$ after infection is significantly greater for $S$. typhimurium compared to either $S$. dublin or S. choleraesuis $(P<0.05)$ (Table 1$)$. Between 1 and $3 \mathrm{~h}$, the recovery of $S$. typhimurium and $S$. dublin decreased slightly $\left(0 \cdot 1-0 \cdot 3 \log _{10}\right.$ c.f.u. $\left.\mathrm{ml}^{-1}\right)$ whereas the recovery of $S$. choleraesuis decreased by almost $1 \cdot 0$ $\log _{10}$ c.f.u. $\mathrm{ml}^{-1}$, and it was recovered in significantly lower numbers than either S. typhimurium or S. dublin $(P<0.05)$. Between 24 and $48 \mathrm{~h}$, the recovery of $S$. typhimurium and S. dublin remained constant, whereas the recovery of $S$. choleraesuis continued to decrease. At $48 \mathrm{~h}$ after infection, $S$. choleraesuis was recovered in significantly lower numbers than either $S$. typhimurium or S. dublin $(P<0.02)$.

The amount of macrophage damage induced during infection with the different serotypes was quantified by measuring the release of LDH. Macrophages infected with $S$. choleraesuis did not release significant amounts of $\mathrm{LDH}$ compared to the uninfected macrophages $(P$ $>0 \cdot 1$ ) at either 3,24 or $48 \mathrm{~h}$ (Table 2). Macrophages infected with S. typhimurium and S. dublin released significantly more LDH than $S$. choleraesuis for several, but not all, of the possible combinations of strains at 24 and $48 \mathrm{~h}(P>0 \cdot 001)$. 
(a) Oral inoculation

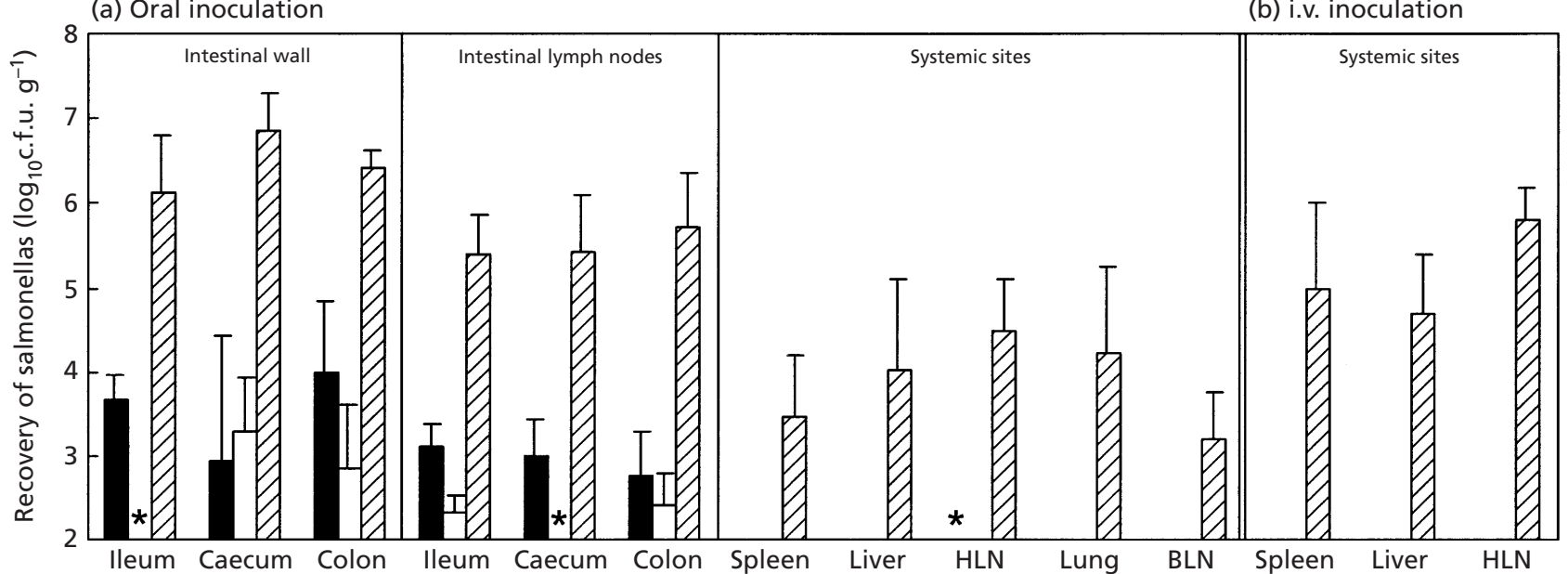

Fig. 2. Recovery of S. typhimurium (filled bars), S. dublin (open bars) and S. choleraesuis (hatched bars) from intestinal and systemic sites following (a) oral inoculation and (b) intravenous inoculation. Each bar is the mean of three pigs, except that for the intravenous inoculation with $S$. typhimurium, which is from only two pigs, and is presented with the standard error of the mean. The limit of accurate quantification was $2.0 \log _{10}$ c.f.u. $\mathrm{g}^{-1}$, and samples that do contain salmonellas, but in numbers too low to quantify, are indicated with an asterisk. HLN, hepatic lymph node; BLN, bronchial lymph node.

Table 1. Recovery of S. typhimurium (ST4/74 and ST12/75), S. dublin (SD2229 and SD3246) and S. choleraesuis (SCSA50 and SCS14/74) from porcine alveolar macrophages

Macrophages were infected with five bacteria per macrophage and the numbers of associated bacteria were determined after incubation for 1, 3, 24 or $48 \mathrm{~h}$. Gentamicin was added at $1 \mathrm{~h}$ after infection to the monolayers used for the 24 and $48 \mathrm{~h}$ time points to prevent the overgrowth of extracellular bacteria. The results are the means of three experiments, each performed in triplicate, and are presented with the standard error of the mean.

\begin{tabular}{|c|c|c|c|c|c|}
\hline \multirow[t]{2}{*}{ Strain } & \multirow{2}{*}{$\begin{array}{l}\text { Inocula } \\
\left(\log _{10} \text { c.f.u. }\right. \\
\left.\mathrm{ml}^{-1}\right)\end{array}$} & \multicolumn{4}{|c|}{ Bacterial recovery $\left(\log _{10}\right.$ c.f.u. $\left.\mathrm{ml}^{-1}\right)$} \\
\hline & & $1 \mathrm{~h}$ & $3 \mathrm{~h}$ & $24 \mathrm{~h}$ & $48 \mathrm{~h}$ \\
\hline ST4/74 & $6 \cdot 5 \pm 0 \cdot 0$ & $5 \cdot 7 \pm 0 \cdot 2$ & $5 \cdot 6 \pm 0 \cdot 3$ & $4 \cdot 9 \pm 0 \cdot 4$ & $4 \cdot 7 \pm 0 \cdot 6$ \\
\hline ST12/75 & $6 \cdot 5 \pm 0 \cdot 0$ & $5 \cdot 8 \pm 0 \cdot 2$ & $5 \cdot 6 \pm 0 \cdot 3$ & $4 \cdot 9 \pm 0 \cdot 5$ & $5 \cdot 1 \pm 0 \cdot 3$ \\
\hline SD2229 & $6 \cdot 5 \pm 0 \cdot 1$ & $5 \cdot 4 \pm 0 \cdot 1$ & $5 \cdot 3 \pm 0 \cdot 2$ & $4 \cdot 5 \pm 0 \cdot 6$ & $4 \cdot 3 \pm 0 \cdot 5$ \\
\hline SD3246 & $6 \cdot 5 \pm 0 \cdot 0$ & $5 \cdot 3 \pm 0 \cdot 0$ & $5 \cdot 0 \pm 0 \cdot 2$ & $4 \cdot 5 \pm 0 \cdot 7$ & $4 \cdot 4 \pm 0 \cdot 3$ \\
\hline SCSA50 & $6 \cdot 6 \pm 0 \cdot 0$ & $5 \cdot 3 \pm 0 \cdot 2$ & $4 \cdot 4 \pm 0 \cdot 3$ & $3 \cdot 7 \pm 0 \cdot 3$ & $3 \cdot 1 \pm 0 \cdot 7$ \\
\hline SCS14/74 & $6 \cdot 4 \pm 0 \cdot 1$ & $5 \cdot 2 \pm 0 \cdot 1$ & $4 \cdot 6 \pm 0 \cdot 2$ & $4 \cdot 0 \pm 0 \cdot 2$ & $3 \cdot 1 \pm 0 \cdot 3$ \\
\hline
\end{tabular}

\section{Release of pro-inflammatory cytokines during infection of porcine alveolar macrophages with different Salmonella serotypes does not correlate with virulence}

The release of the pro-inflammatory cytokines IL-1, TNF- $\alpha$ and IL- 6 from porcine alveolar macrophages infected with different Salmonella serotypes was assessed by biological assays using the same infected macrophages as used in the persistence and lysis assay. Uninfected macrophage monolayers released little IL-1, TNF- $\alpha$ or IL- 6 over the time course of the assay (Table $3)$. Macrophages infected with viable bacteria released IL-1 and TNF- $\alpha$ at all three time points and increasing amounts of IL- 6 over the three time points. There were no significant differences between any of the strains $(P$ $>0.05)$ with the exception of the release of TNF- $\alpha$ by ST4/74 and ST12/75 at $48 \mathrm{~h}$ after infection $(P>0 \cdot 02)$. The general lack of difference between the serotypes was not due to the amount of cytokines being outside the range of detection of the assay as assessed by testing serial dilutions of all of the samples (data not shown). Macrophages infected with heat-inactivated bacteria released low or undetectable levels of all three cytokines at 24 and $48 \mathrm{~h}$ after infection. At $3 \mathrm{~h}$ after infection, IL1 and TNF- $\alpha$ were released, but in lower amounts than from macrophages infected with viable bacteria $(P$ $<0 \cdot 01)$, with the exception of TNF- $\alpha$ release by SCSA50 
Table 2. Damage to porcine alveolar macrophages during infection with $S$. typhimurium (ST4/74 and ST12/75), S. dublin (SD2229 and SD3246) or S. choleraesuis (SCSA50 and SCS14/74)

Damage was estimated by measuring the amount of LDH released by the infected macrophages into the overlying tissue culture medium and is expressed as a percentage compared to the LDH released following detergent treatment of macrophage monolayers. Each result represents the mean of three separate experiments, with each experiment performed in triplicate, and is presented with the standard error of the mean.

\begin{tabular}{|c|c|c|c|}
\hline \multirow[t]{2}{*}{ Strain } & \multicolumn{3}{|c|}{ Macrophage damage (\%) } \\
\hline & $3 \mathrm{~h}$ & $24 \mathrm{~h}$ & $48 \mathrm{~h}$ \\
\hline ST4/74 & $18 \cdot 6 \pm 2 \cdot 8$ & $39 \cdot 6 \pm 3 \cdot 7$ & $75 \cdot 2 \pm 6 \cdot 2$ \\
\hline ST12/75 & $11 \cdot 8 \pm 0 \cdot 8$ & $25 \cdot 7 \pm 4 \cdot 4$ & $58 \cdot 7 \pm 6 \cdot 3$ \\
\hline SD2229 & $12 \cdot 4 \pm 1 \cdot 1$ & $24 \cdot 8 \pm 5 \cdot 0$ & $58 \cdot 8 \pm 5 \cdot 8$ \\
\hline SD3246 & $12 \cdot 7 \pm 0 \cdot 8$ & $28 \cdot 5 \pm 7 \cdot 1$ & $47 \cdot 0 \pm 3 \cdot 3$ \\
\hline SCSA50 & $13 \cdot 0 \pm 3 \cdot 0$ & $11 \cdot 7 \pm 3 \cdot 2$ & $37 \cdot 1 \pm 7 \cdot 7$ \\
\hline SCS14/74 & $8 \cdot 6 \pm 1 \cdot 0$ & $11 \cdot 7 \pm 2 \cdot 5$ & $39 \cdot 3 \pm 7 \cdot 0$ \\
\hline Uninfected & $9 \cdot 3 \pm 1 \cdot 7$ & $8 \cdot 1 \pm 0 \cdot 9$ & $39 \cdot 7 \pm 4 \cdot 3$ \\
\hline
\end{tabular}

$(P>0 \cdot 05)$. Heat-inactivated ST4/74 and SD2229 released more IL-1 than heat-inactivated SCSA50 $(P$ $<0.001)$, and there was no difference in the amount of
TNF- $\alpha$ released by the different heat-inactivated serotypes.

\section{Salmonella serotypes do not induce porcine alveolar macrophage lysis by apoptosis}

The mechanism of Salmonella-induced macrophage lysis was investigated by examining macrophage DNA by electrophoresis for the characteristic laddering pattern associated with apoptosis. Macrophage DNA was isolated at 3, 24 and $48 \mathrm{~h}$ after infection. There was no increase in DNA fragmentation following infection with any Salmonella serotype, at any time point compared to the uninfected control (Fig. 3). Opsonization of the bacteria with autologous sera before infection did not affect the DNA morphology. DNA from macrophages incubated for $24 \mathrm{~h}$ with the apoptosis-inducing agent actinomycin D-mannitol exhibited the laddering pattern associated with apoptosis.

The ultrastructure of porcine alveolar macrophages $24 \mathrm{~h}$ after infection with Salmonella serotypes was examined by transmission electron microscopy. In uninfected monolayers, the large majority of macrophages appeared healthy (Fig. 4a). Infection with $S$. dublin and $S$. typhimurium resulted in loss of pseudopodia and an increase in cell size (Fig. 4b). Opsonization of bacteria before infection had little or no effect on the appearance of the macrophages. Incubation with actinomycin $\mathrm{D}^{-}$ mannitol for $24 \mathrm{~h}$ resulted in cell shrinkage, formation

Table 3. Release of pro-inflammatory cytokines from porcine alveolar macrophages during infection with viable or heat-inactivated Salmonella serotypes

Results are the mean of duplicate bioassays of conditioned supernatants. Supernatants were prepared in triplicate from three independent macrophage infection experiments and are presented with the standard error of the mean.

\begin{tabular}{|c|c|c|c|c|c|c|c|c|c|}
\hline & \multicolumn{3}{|c|}{ IL-1* } & \multicolumn{3}{|c|}{ TNF- $\alpha \dagger$} & \multicolumn{3}{|c|}{ IL-6‡ } \\
\hline & $3 \mathrm{~h} \mathbb{S}$ & $24 \mathrm{~h} \|$ & $48 \mathrm{~h} \|$ & $3 \mathrm{~h}$ & $24 \mathrm{~h}$ & $48 \mathrm{~h}$ & $3 \mathrm{~h}$ & $24 \mathrm{~h}$ & $48 \mathrm{~h}$ \\
\hline \multicolumn{10}{|c|}{ Viable bacteria } \\
\hline ST4/74 & $69 \cdot 9 \pm 2 \cdot 8$ & $62 \cdot 1 \pm 6 \cdot 0$ & $79 \cdot 1 \pm 6 \cdot 1$ & $78 \cdot 3 \pm 1 \cdot 1$ & $63 \cdot 7 \pm 7 \cdot 0$ & $41 \cdot 1 \pm 3 \cdot 4$ & $6 \cdot 3 \pm 0 \cdot 5$ & $14 \cdot 7 \pm 1 \cdot 1$ & $22 \cdot 5 \pm 2 \cdot 0$ \\
\hline ST12/75 & $69 \cdot 8 \pm 3 \cdot 4$ & $57 \cdot 0 \pm 6 \cdot 0$ & $70 \cdot 7 \pm 7 \cdot 5$ & $77 \cdot 0 \pm 2 \cdot 8$ & $63 \cdot 3 \pm 6 \cdot 4$ & $40 \cdot 8 \pm 5 \cdot 7$ & $5 \cdot 6 \pm 0 \cdot 2$ & $15 \cdot 3 \pm 1 \cdot 5$ & $18 \cdot 3 \pm 1 \cdot 3$ \\
\hline SD2229 & $71 \cdot 6 \pm 3 \cdot 0$ & $60 \cdot 4 \pm 4 \cdot 8$ & $80 \cdot 1 \pm 5 \cdot 2$ & $82.4 \pm 3.6$ & $74 \cdot 0 \pm 5 \cdot 3$ & $58 \cdot 2 \pm 2 \cdot 4$ & $6 \cdot 2 \pm 0 \cdot 4$ & $14 \cdot 8 \pm 2 \cdot 0$ & $24 \cdot 1 \pm 1 \cdot 9$ \\
\hline SD3246 & $72 \cdot 4 \pm 3 \cdot 3$ & $62 \cdot 0 \pm 4 \cdot 8$ & $76 \cdot 3 \pm 8 \cdot 7$ & $73 \cdot 4 \pm 4 \cdot 9$ & $74 \cdot 6 \pm 5 \cdot 0$ & $55 \cdot 3 \pm 2 \cdot 1$ & $5 \cdot 4 \pm 0 \cdot 3$ & $15 \cdot 9 \pm 2 \cdot 0$ & $17 \cdot 2 \pm 1 \cdot 7$ \\
\hline SCSA50 & $71 \cdot 5 \pm 2 \cdot 0$ & $60 \cdot 1 \pm 5 \cdot 5$ & $77 \cdot 0 \pm 4 \cdot 8$ & $73 \cdot 8 \pm 4 \cdot 9$ & $70 \cdot 8 \pm 5 \cdot 6$ & $53 \cdot 9 \pm 2 \cdot 2$ & $8 \cdot 5 \pm 0 \cdot 8$ & $18 \cdot 4 \pm 1 \cdot 6$ & $26 \cdot 7 \pm 2 \cdot 3$ \\
\hline SCS14/74 & $66 \cdot 0 \pm 2 \cdot 5$ & $57 \cdot 4 \pm 6 \cdot 6$ & $68 \cdot 0 \pm 7 \cdot 6$ & $71 \cdot 3 \pm 7 \cdot 7$ & $64 \cdot 4 \pm 5 \cdot 8$ & $59 \cdot 9 \pm 2 \cdot 6$ & $7 \cdot 3 \pm 0 \cdot 5$ & $17 \cdot 2 \pm 1 \cdot 3$ & $23 \cdot 6 \pm 2 \cdot 1$ \\
\hline \multicolumn{10}{|c|}{ Heat-inactivated bacteria } \\
\hline ST4/74 & $52 \cdot 4 \pm 5 \cdot 8$ & $19 \cdot 2 \pm 4 \cdot 5$ & $28 \cdot 8 \pm 3 \cdot 5$ & $51 \cdot 3 \pm 8 \cdot 0$ & $16 \cdot 3 \pm 5 \cdot 9$ & $1 \cdot 7 \pm 1 \cdot 1$ & $5 \cdot 0 \pm 0 \cdot 3$ & $8 \cdot 1 \pm 0 \cdot 6$ & $10 \cdot 4 \pm 1 \cdot 7$ \\
\hline SD2229 & $50 \cdot 2 \pm 5 \cdot 2$ & $14 \cdot 7 \pm 3 \cdot 0$ & $29 \cdot 0 \pm 5 \cdot 7$ & $40 \cdot 9 \pm 9 \cdot 5$ & $4 \cdot 5 \pm 2 \cdot 6$ & $3 \cdot 4 \pm 3 \cdot 9$ & $7 \cdot 1 \pm 0 \cdot 5$ & $8 \cdot 6 \pm 0 \cdot 8$ & $10 \cdot 4 \pm 2 \cdot 3$ \\
\hline SCSA50 & $35 \cdot 7 \pm 4 \cdot 9$ & $18 \cdot 4 \pm 6 \cdot 2$ & $28 \cdot 3 \pm 4 \cdot 0$ & $65 \cdot 6 \pm 7 \cdot 1$ & $24 \cdot 0 \pm 8 \cdot 8$ & $3 \cdot 5 \pm 2 \cdot 3$ & $5 \cdot 8 \pm 0 \cdot 3$ & $8 \cdot 1 \pm 1 \cdot 0$ & $6 \cdot 8 \pm 0 \cdot 9$ \\
\hline Uninfected & $10 \cdot 1 \pm 2 \cdot 7$ & $18 \cdot 1 \pm 6 \cdot 7$ & $16 \cdot 5 \pm 3 \cdot 7$ & $-1 \cdot 7 \pm 5 \cdot 4$ & $20 \cdot 4 \pm 11 \cdot 7$ & $24 \cdot 1 \pm 13 \cdot 3$ & $4 \cdot 2 \pm 0 \cdot 3$ & $7 \cdot 6 \pm 0 \cdot 8$ & $7 \cdot 5 \pm 1 \cdot 6$ \\
\hline
\end{tabular}

*IL-1 bio-activity (\% growth inhibition) of conditioned macrophage supernatant on A375 cells.

†TNF- $\alpha$ bio-activity (\% cytotoxicity) of conditioned supernatant on WEHI 164 clone 13 cells.

$\ddagger$ IL-6 bio-activity (cell growth measured by uptake of tritiated thymidine; $\times 1000$ c.p.m.) of conditioned supernatant on 7 TD1 cells. $\$$ Macrophages were infected for $3 \mathrm{~h}$ and supernatants were collected.

$\|$ Macrophages were infected for 24 or $48 \mathrm{~h}$, with the addition of gentamicin $1 \mathrm{~h}$ after infection to prevent bacterial overgrowth in the media, and supernatants were collected. 


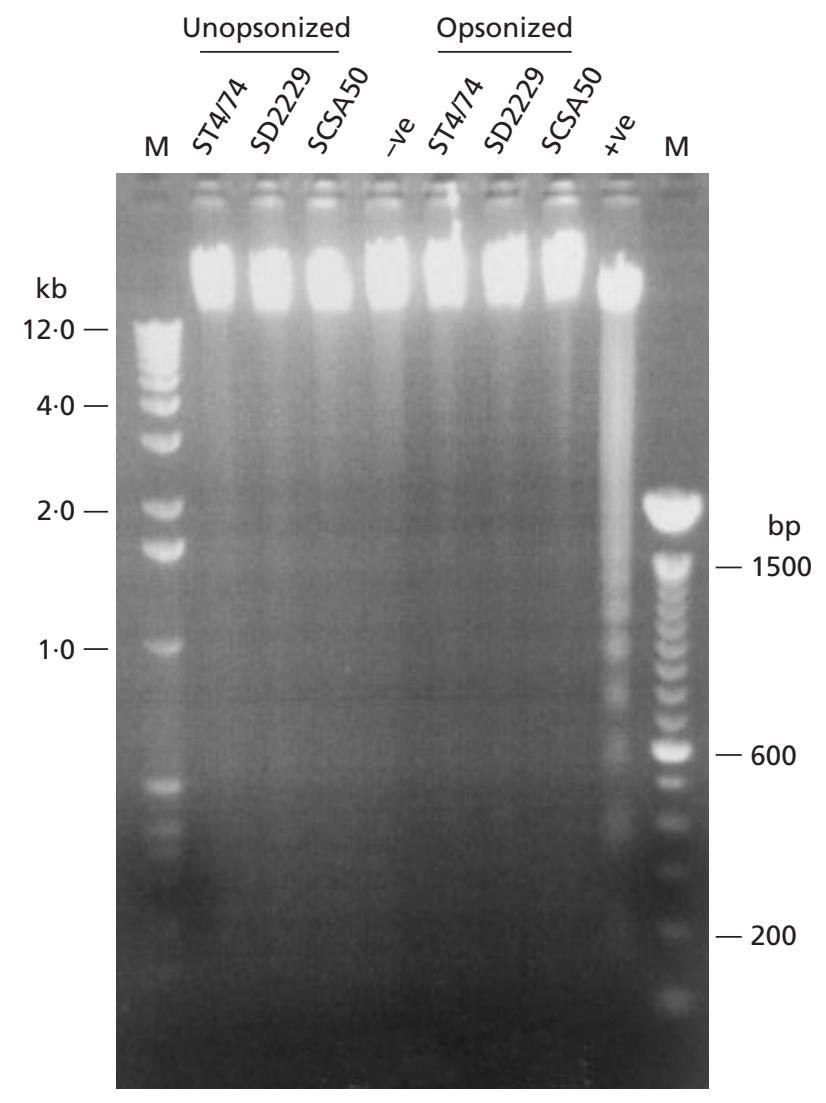

Fig. 3. Characterization of DNA extracted from porcine alveolar macrophages infected for $24 \mathrm{~h}$ with $S$. typhimurium ST4/74, S. dublin SD2229 or S. choleraesuis SCSA50. Uninfected macrophages were used as the negative control ( - ve) and macrophages incubated with actinomycin D-mannitol for $24 \mathrm{~h}$ were used as the positive control $(+v e)$. This is a representative result from a total of three separate experiments. The original photograph of the gel was scanned using a Kodak DCS420 digital camera and the contrast of the image was adjusted using Adobe Photoshop 3.0.

of apoptotic bodies and marginalization of condensed chromatin (Fig. 4c).

\section{DISCUSSION}

The apparent specificity of a given Salmonella serotype for a particular host will be influenced by both the likelihood of natural exposure and the degree of bacterial virulence for the host. Therefore, virulence following experimental infection may not necessarily correlate with epidemiological data on serotype-host specificity. For example, S. choleraesuis can infect pigs, cattle, mice, rats, guinea pigs and rabbits after experimental inoculation (Williams Smith \& Halls, 1966, 1968; Reed et al., 1986; Nnalue, 1991; Nnalue et al., 1992; Barrow et al., 1994), but is generally only associated with natural infection of pigs, and infrequently of man. In addition, natural infection may occur with a 'non-specific' combination of serotype and host as happened during an S. dublin epidemic in cattle in England and Wales during the late 1960s, in which
S. dublin was also associated with $19 \%$ of porcine Salmonella incidents, compared to a mean of only $0-1 \%$ at other times (Sojka \& Field, 1970; Anonymous, 1978-1998). In contrast, with some combinations of serotype and host there is a strong correlation between virulence following experimental infection and serotypehost specificity. For example, the inability to infect several laboratory animal species with $S$. typhi and Salmonella gallinarum correlates with their very restricted host range in nature. Therefore, studies of host specificity need to consider the virulence of each serotype-host combination, and extrapolation of results from one combination to another is not possible.

In the present study, the relative virulence of $S$. choleraesuis, S. typhimurium and S. dublin for pigs was determined. Pigs can be experimentally infected with $S$. typhimurium and $S$. choleraesuis by both oral and intranasal inoculation (Reed et al., 1986; Fedorka-Cray et al., 1995; Gray et al., 1995), and infection via other routes, independent of the gastro-intestinal tract, may also be possible. The relative importance of different routes of natural infection in pigs is not known, and therefore the virulence of the three serotypes was assessed following both oral and intravenous inoculation. S. choleraesuis induced severe symptoms and infected systemic tissues in high numbers, S. typhimurium induced an acute, self-limiting enteritis and $S$. dublin was of low virulence. All three strains used to infect pigs are highly virulent for mice (Baird et al., 1985; Plested, 1995; Guilloteau et al., 1996; A. V. Gautier \& T. S. Wallis, unpublished data). These results confirm the association of $S$. choleraesuis with systemic disease in pigs, and the general lack of association of $S$. dublin with porcine salmonellosis. They also suggest that the mechanism of serotype-host specificity in pigs does not depend on intestinal colonization and translocation, since $S$. choleraesuis was the most virulent serotype by both routes of inoculation. This supports the recent observation that serotype-host specificity of $S$. dublin and $S$. choleraesuis does not correlate with intestinal invasion of bovine or porcine intestinal mucosa (Bolton et al., 1999).

For many years it has been widely accepted that a key stage in the pathogenesis of Salmonella is its persistence within cells of the reticuloendothelial system. For example, susceptibility of mice to salmonellosis correlates with net growth of $S$. typhimurium within isolated macrophages (reviewed by Dunlap et al., 1994) and Salmonella mutants that are unable to survive in murine macrophages have reduced virulence in mice (Fields et al., 1986; Libby et al., 1994; Hensel et al., 1998). However, several independent groups have recently reported that Salmonella is highly cytolytic for macrophages (Guilloteau et al., 1996; Chen et al., 1996; Lindgren et al., 1996), suggesting that the contribution of macrophage infection to pathogenesis is more complex than previously believed. We have therefore investigated several parameters of the interaction of our strains of defined porcine virulence with primary porcine alveolar macrophages. This macro- 

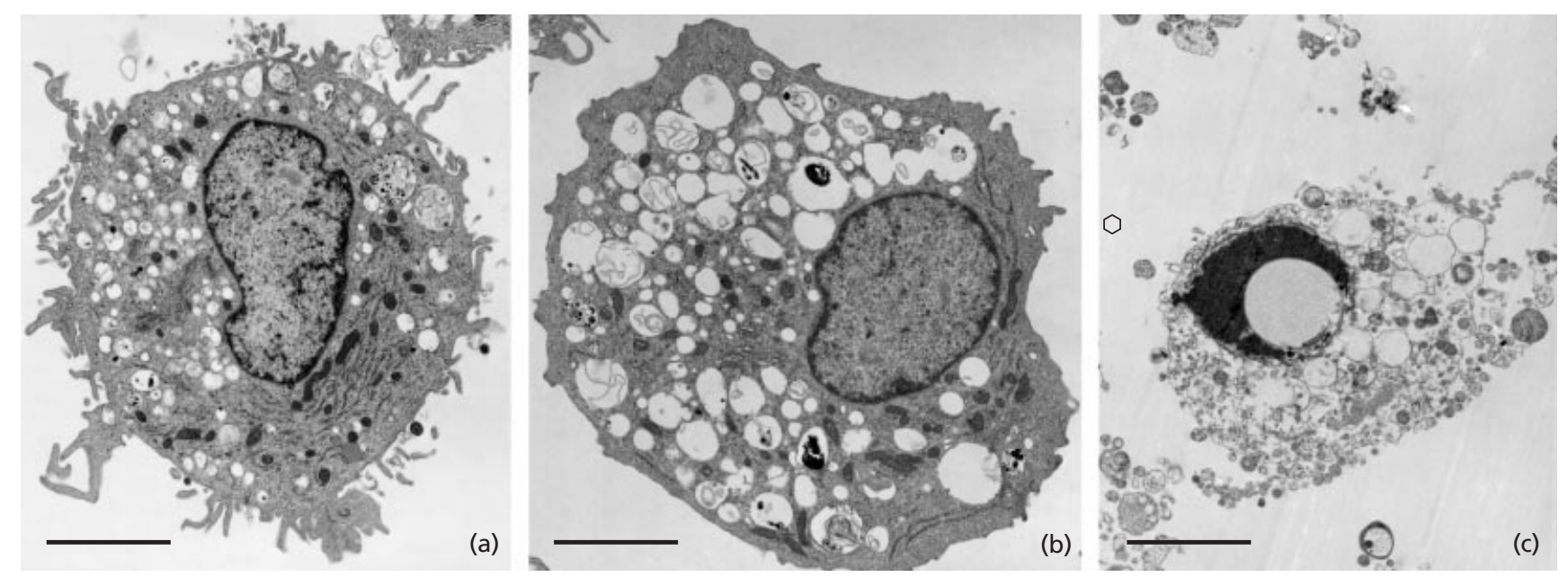

Fig. 4. Transmission electron microscopy of porcine alveolar macrophages either (a) without treatment, (b) $24 \mathrm{~h}$ after infection with S. typhimurium ST4/74 or (c) $24 \mathrm{~h}$ after incubation with actinomycin D-mannitol. See Results for description of morphological changes. This is a representative result from three separate experiments. Micrograph negatives were scanned using a linotype Saphir flatbed scanner and the image was converted to positive and the contrast was adjusted using Adobe Photoshop 3.0. Bar, $4 \mu \mathrm{m}$.

phage population has been relatively well characterized and is phagocytic, bactericidal, produces pro-inflammatory mediators, expresses several surface receptors typical of other macrophages and is easy to isolate in high numbers and purity (reviewed by Van Reeth \& Adair, 1997). In addition, in pigs the lungs are an important site for clearance of bacteria and particulates from blood (Winkler, 1988), and pneumonia is a common feature of $S$. choleraesuis infection of pigs and therefore it is probable that $S$. choleraesuis interacts with alveolar macrophages during pathogenesis. However, porcine alveolar macrophages may differ in some of their properties to tissue macrophages in other systemic organs, and therefore may not necessarily accurately model the interaction of Salmonella with macrophages in the porcine liver and spleen. Macrophage-derived cell lines were not used because they are dramatically more permissive for uptake and/or intracellular replication of Salmonella serotypes compared to primary macrophages (Buchmeier \& Heffron, 1989; Vladoianu et al., 1990) and would appear to be inherently unsuitable for such studies. For example, in murine macrophage-like cells, some studies find no difference between $S$. typhimurium and $S$. typhi (Vladoianu et al., 1990; Hirose et al., 1997), and others report differences in either uptake, but not persistence (Pascopella et al., 1995), or persistence, but not uptake (Schwan \& Kopecko, 1997). In the present study, the majority of macrophage experiments were performed using non-opsonized bacteria, since preliminary experiments showed that opsonization was not a prerequisite for efficient phagocytosis and did not influence subsequent persistence, as has sometimes been reported for other serotype-host combinations (Ishibashi \& Arai, 1996).

The relative persistence of S. typhimurium, S. dublin and $S$. choleraesuis in porcine alveolar macrophages did not directly correlate with their virulence in pigs. $S$. typhimurium and S. dublin, but not S. choleraesuis, induced damage to the macrophage plasma membrane. This may allow gentamicin to enter the macrophage and kill intracellular bacteria, and therefore the difference in recovery of $S$. typhimurium and S. dublin compared to $S$. choleraesuis is probably greater than that measured by viable counts. These persistence data appear to be in contrast to those obtained with murine macrophages, from which it was concluded that the persistence of $S$. typhimurium and S. typhi directly correlated with their host specificity (Lissner et al., 1985; Vladoianu et al., 1990; Alpuche-Aranda et al., 1995; Ishibashi \& Arai, 1996; Schwan \& Kopecko, 1997). However, S. typhimurium can persist relatively well not only in murine macrophages, but also in human-monocyte-derived macrophages, chicken peritoneal exudate cells and porcine alveolar macrophages (Vladoianu et al., 1990; Alpuche-Aranda et al., 1995; Ishibashi \& Arai, 1996; M. S. Chadfield \& J. E. Olsen, personal communication; this study). Taking these results together, there is a direct correlation between $S$. typhimurium persistence within macrophages and its ability to cause disease in a wide range of animals.

Macrophages are important in the regulation of the host's immune response to infection through the release of pro-inflammatory cytokines and other mediators of inflammation. This contributes to the mobilization of host defences and recruitment of other immune cells required for control of an infection, but may also exacerbate some aspects of disease (reviewed by Murtaugh et al., 1996). The release of three proinflammatory cytokines by macrophages infected with different serotypes was quantified to determine whether this correlates with the different disease syndromes. 
Infection of porcine macrophages with all three serotypes resulted in a rapid and sustained release of IL-1 and TNF- $\alpha$ activity and a more gradual increase in the release of IL-6 activity. This is despite differences in the numbers of associated bacteria and the magnitude of macrophage damage. Therefore, the ability of different Salmonella serotypes to induce the release of proinflammatory cytokines from macrophages in vitro does not correlate with serotype-host specificity. Heat-inactivated bacteria were included in the cytokine assays to determine if any differences between the Salmonella serotypes were related to differences in surface antigens such as LPS. Although it turned out that this control was not necessary, it did reveal a difference in cytokine release induced by viable and killed bacteria. This supports a previous report showing differences in IL-12 induction in murine macrophages by viable and killed $S$. dublin (Chong et al., 1996) and is relevant to the design of vaccines for salmonellosis.

Salmonella-induced macrophage damage has been reported to occur in vivo in both mice and pigs (Baskerville et al., 1972; Richter-Dahlfors et al., 1997). However, in the present study there was no direct correlation between Salmonella-induced macrophage damage in vitro and virulence of the same strains in pigs. Furthermore, macrophage damage induced by S. typhimurium and $S$. dublin did not have any of the typical characteristics of apoptosis (reviewed by Allen et al., 1997; McConkey, 1998), which is in contrast to the conclusions of several other studies on Salmonella-induced macrophage damage (Chen et al., 1996; Monack et al., 1996; Hersh et al., 1999). This is unlikely to be due to the use of different cell types or bacterial strains, since we have previously shown that Salmonella can damage macrophages by a non-apoptotic mechanism using both an immortalized macrophage-like cell line and alveolar macrophages isolated from cattle following infection with several commonly studied S. typhimurium strains (Watson et al., 2000). In addition, opsonization of the bacteria did not alter the mechanism of macrophage damage. The induction of macrophage damage by a non-apoptotic mechanism is consistent with several features of Salmonella pathogenesis. For example, Salmonella infection of the intestines is associated with a large inflammatory response whereas apoptosis is generally believed to limit inflammation (reviewed by Savill, 1997). In addition, there seems little benefit to an intracellular pathogen in inducing apoptosis in its host cell, since the pathogen may then become trapped within the apoptotic cell. This is analogous to many viral infections, in which apoptosis of the host cell is blocked to facilitate viral replication and spread (reviewed by Tschopp et al., 1998). Conversely, the induction of apoptosis by predominately extracellular pathogens, such as Yersinia spp., may prevent phagocytosis and subsequent bacterial killing (Monack et al., 1997; Ruckdeschel et al., 1997).

This study reports on several in vitro assays which model the interaction of Salmonella and macrophages during pathogenesis with respect to host specificity.
Although persistence within macrophages correlated with the broad host specificity of $S$. typhimurium, there were no direct correlations between persistence within macrophages, induction of cytokine release by macrophages or the severity or nature of macrophage damage with Salmonella serotype specificity for pigs. There is relatively little known about the interaction of Salmonella and macrophages in vivo and it is therefore possible that such in vitro assays do not accurately model the interaction in vivo. Further work will address this problem by characterizing the interaction between Salmonella and host cells in intestinal and systemic tissues following experimental infection of natural host animal species.

\section{ACKNOWLEDGEMENTS}

This work was supported by the Ministry for Agriculture Food and Fisheries, the Biological and Biotechnological Science Research Council and the European Union. The authors are grateful to B. Villarreal-Ramos, B. Collins and P. Kaiser for advice on performing cytokine bioassays, to A. P. Bland and T. Smith for electron microscopy analysis and to S. Kidd for supplying epidemiological data.

\section{REFERENCES}

Allen, R. T., Hunter, W. J., III \& Agrawal, D. K. (1997). Morphological and biochemical characterization and analysis of apoptosis. J Pharmacol Toxicol Methods 37, 215-228.

Alpuche-Aranda, C. M., Berthiaume, E. P., Mock, B., Swanson, J. A. \& Miller, S. I. (1995). Spacious phagosome formation within mouse macrophages correlates with Salmonella serotype pathogenicity and host susceptibility. Infect Immun 63, 4456-4462.

Anonymous (1978-1998). Annual reports on Salmonella in livestock production. Veterinary Laboratories Agency, Ministry of Agriculture, Fisheries and Food, UK.

Baird, G. D., Manning, E. J. \& Jones, P. W. (1985). Evidence for related virulence sequences in plasmids of Salmonella dublin and Salmonella typhimurium. J Gen Microbiol 131, 1815-1823.

Barrow, P. A., Huggins, M. B. \& Lovell, M. A. (1994). Host specificity of Salmonella infection in chickens and mice is expressed in vivo primarily at the level of the reticuloendothelial system. Infect Immun 62, 4602-4610.

Baskerville, A., Dow, C., Curran, W. L. \& Hanna, J. (1972). Ultrastructure of phagocytosis of Salmonella cholerae-suis by pulmonary macrophages in vivo. Br J Exp Pathol 53, 641-647.

Berends, B. R., van Knapen, F., Snijers, J. M. \& Mossel, D. A. (1997). Identification and quantification of risk factors regarding Salmonella spp. on pork carcasses. Int J Food Microbiol 36, 199-206.

Bolton, A. J., Osborne, M. P., Wallis, T. S. \& Stephen, J. (1999). Interaction of Salmonella choleraesuis, Salmonella dublin and Salmonella typhimurium with porcine and bovine terminal ileum in vivo. Microbiology 145, 2431-2441.

Buchmeier, N. A. \& Heffron, F. (1989). Intracellular survival of wild-type Salmonella typhimurium and macrophage-sensitive mutants in diverse populations of macrophages. Infect Immun 57, $1-7$.

Cendan, J. C., Moldawer, L. L., Souba, W. W., Copeland, E. M. \& Lind, D. S. (1994). Endotoxin-induced nitric oxide production in pulmonary artery endothelial cells is regulated by cytokines. Arch Surg 129, 1296-1300. 
Chen, L. M., Kaniga, K. \& Galán, J. E. (1996). Salmonella spp. are cytotoxic for cultured macrophages. Mol Microbiol 21, 1101-1115.

Chong, C., Bost, K. L. \& Clements, J. D. (1996). Differential production of interleukin-12 mRNA by murine macrophages in response to viable or killed Salmonella spp. Infect Immun 64, 1154-1160.

Dunlap, N. E., Benjamin, W. H., Jr \& Briles, D. E. (1994). The intracellular nature of Salmonella infection during the early stages of mouse typhoid. Immunol Ser 60, 303-312.

Espevik, T. \& Nissen-Meyer, J. (1986). A highly sensitive cell line, WEHI 164 clone 13, for measuring cytotoxic factor/tumour necrosis factor from human monocytes. J Immunol Methods 95, 99-105.

Fedorka-Cray, P. J., Kelley, L. C., Stabel, T. J., Cray, J. T. \& Laufer, J. A. (1995). Alternate routes of invasion may affect pathogenesis of Salmonella typhimurium in swine. Infect Immun 63, 2658-2664.

Fields, P. I., Swanson, R. V., Haidaris, C. G. \& Heffron, F. (1986). Mutants of Salmonella typhimurium that cannot survive within the macrophage are avirulent. Proc Natl Acad Sci USA 83, 5189-5193.

Frank, D. E., Ahrens, F. A. \& Kramer, T. T. (1996). Cytokine release by porcine livers perfused with lipopolysaccharide or live Salmonella choleraesuis. Am J Vet Res 57, 472-476.

Ganter, M. \& Hensel, A. (1997). Cellular variables in bronchoalveolar lavage fluids (BALF) in selected healthy pigs. Res Vet Sci 63, 215-217.

Gray, J. T., Fedorka-Cray, P. J., Stabel, T. J. \& Ackermann, M. R. (1995). Influence of inoculation route on the carrier state of Salmonella choleraesuis in swine. Vet Microbiol 47, 43-59.

Guilloteau, L. A., Wallis, T. S., Gautier, A. V., Maclntyre, S., Platt, D. J. \& Lax, A. J. (1996). The Salmonella virulence plasmid enhances Salmonella-induced lysis of macrophages and influences inflammatory responses. Infect Immun 64, 3385-3393.

Hensel, M., Shea, J. E., Waterman, S. R. \& 7 other authors (1998). Genes encoding putative effector proteins of the type III secretion system of Salmonella pathogenicity island 2 are required for bacterial virulence and proliferation in macrophages. Mol Microbiol 30, 163-174.

Hersh, D., Monack, D. M., Smith, M. R., Ghori, N., Falkow, S. \& Zychlincky, A. (1999). The Salmonella invasin SipB induces macrophage apoptosis by binding to caspase-1. Proc Natl Acad Sci USA 96, 2396-2401.

Hirose, K., Ezaki, T., Miyake, M., Li, T., Khan, A. Q., Kawamura, Y., Yokoyama, H. \& Takami, T. (1997). Survival of Vi-capsulated and Vi-deleted Salmonella typhi strains in cultured macrophages expressing different levels of CD14 antigen. FEMS Microbiol Lett 147, 259-265.

Ishibashi, Y. \& Arai, T. (1996). A possible mechanism for hostspecific pathogenesis of Salmonella serovars. Microb Pathog 21, 435-446.

Jones, P. W. (1975). The effect of storage in slurry on the virulence of Salmonella dublin. J Hyg 74, 64-70.

Kim, K. W., Wierda, W. G. \& Kim, Y. B. (1991). Immobilised IgG immune complex induces secretion of tumour necrosis factor- $\alpha$ by porcine alveolar macrophages. Am J Respir Cell Mol Biol 5, 249-255.

Libby, S. J., Goebel, W., Ludwig, A., Buchmeier, N., Bowe, F., Fang, F. C., Guiney, D. G., Songer, J. G. \& Heffron, F. (1994). A cytolysin encoded by Salmonella is required for survival within macrophages. Proc Natl Acad Sci USA 91, 489-493.
Lindgren, S. W., Stojiljkovic, I. \& Heffron, F. (1996). Macrophage killing is an essential virulence mechanism of Salmonella typhimurium. Proc Natl Acad Sci USA 93, 4197-4201.

Lissner, C. R., Weinstein, D. L. \& O'Brien, A. D. (1985). Mouse chromosome 1 Ity locus regulates microbicidal activity of isolated peritoneal macrophages against a diverse group of intracellular and extracellular bacteria. J Immunol 135, 544-547.

McConkey, D. J. (1998). Biochemical determinants of apoptosis and necrosis. Toxicol Lett 99, 157-168.

Monack, D. M., Raupach, B., Hromockyj, A. E. \& Falkow, S. (1996). Salmonella typhimurium invasion induces apoptosis in infected macrophages. Proc Natl Acad Sci USA 93, 9833-9838.

Monack, D. M., Mecsas, J., Ghori, N. \& Falkow, S. (1997). Yersinia signals macrophages to undergo apoptosis and YopJ is necessary for this cell death. Proc Natl Acad Sci USA 94, 10385-10390.

Murtaugh, M. P., Baarsch, M. J., Zhou, Y., Scamurra, R. W. \& Lin, G. (1996). Inflammatory cytokines in animal health and disease. Vet Immunol Immunopathol 54, 45-55.

Nakai, S., Mizuno, K., Kaneta, M. \& Hirai, Y. (1988). A simple, sensitive bioassay for the detection of interleukin-1 using human melanoma A375 cell line. Biochem Biophys Res Commun 154, 1189-1196.

Nnalue, N. A. (1991). Relevance of inoculation route to virulence of three Salmonella spp. strains in mice. Microb Pathol 11, 11-18.

Nnalue, N. A., Shnyra, A., Hultenby, K. \& Lindberg, A. A. (1992). Salmonella choleraesuis and Salmonella typhimurium associated with liver cells after intravenous inoculation of rats are localised in kupffer cells and multiply intracellularly. Infect Immun 60, 2758-2768.

Pascopella, L., Raupach, B., Ghori, N., Monack, D., Falkow, S. \& Small, P. L. C. (1995). Host restriction phenotypes of Salmonella typhi and Salmonella gallinarum. Infect Immun 63, 4329-4335.

Plested, J. S. (1995). Identification of the antigens of salmonellas involved in the protective immune response. $\mathrm{PhD}$ thesis, University of Reading, UK.

Reed, W. M., Olander, H. J. \& Thacker, H. L. (1986). Studies on the pathogenesis of Salmonella typhimurium and Salmonella choleraesuis var kunzendorf infection in weanling pigs. Am J Vet Res 47, 75-83.

Richter-Dahlfors, A., Buchan, M. J. \& Finlay, B. B. (1997). Murine salmonellosis studies by confocal microscopy: Salmonella typhimurium resides intracellularly inside macrophages and exerts a cytotoxic effect on phagocytes in vivo. J Exp Med 186, 569-580.

Ruckdeschel, K., Roggenkamp, A., Lafont, V., Mangeat, P., Heesemann, J. \& Rouot, B. (1997). Interaction of Yersinia enterocolitica with macrophages leads to macrophage cell death through apoptosis. Infect Immun 65, 4813-4821.

Savill, J. (1997). Apoptosis in resolution of inflammation. J Leukoc Biol 61, 375-380.

Schwan, W. R. \& Kopecko, D. J. (1997). Serovar specific differences in Salmonella survival within macrophage cells. Adv Exp Med Biol 412, 277-278.

Sizemore, D. R., Elinghorst, E. A., Eck, L. C., Branstrom, A. A., Hoover, D. L., Warren, R. L. \& Rubin, F. A. (1997). Interaction of Salmonella typhi strains with cultured human monocyte-derived macrophages. Infect Immun 65, 309-312.

Sojka, W. J. \& Field, H. I. (1970). Salmonellosis in England and Wales, 1958-1967. Vet Bull 40, 515-531.

Tschopp, J., Thome, M., Hofmann, K. \& Meinl, E. (1998). The fight of viruses against apoptosis. Curr Opin Genet Dev 8, 82-87. 
Van Reeth, K. \& Adair, B. (1997). Macrophages and respiratory viruses. Pathol Biol 45, 184-192.

Van Snick, J., Cayphas, S., Vink, A., Uyttenhove, C., Coulie, P. G., Rubira, M. R. \& Simpson, R. J. (1986). Purification and NH2terminal amino acid sequence of a T-cell-derived lymphokine with growth factor activity for B-cell hybridomas. Proc Natl Acad Sci USA 83, 9679-9683.

Vladoianu, I.-R., Chang, H. R. \& Pechère, J.-C. (1990). Expression of host resistance to Salmonella typhi and Salmonella typhimurium: bacterial survival within macrophages of murine and human origin. Microb Pathog 8, 83-90.

Wallis, T. S., Paulin, S. M., Plested, J. S., Watson, P. R. \& Jones, P. W. (1995). The Salmonella dublin virulence plasmid mediates systemic but not enteric phases of salmonellosis in cattle. Infect Immun 63, 2755-2761.

Watson, P. R., Galyov, E. E., Paulin, S. M., Jones, P. W. \& Wallis, T. S. (1998). Mutation of $i n v H$, but not stn reduced Salmonellainduced enteritis in cattle. Infect Immun 66, 1432-1438.

Watson, P. R., Gautier, A. V., Paulin, S. M., Bland, A. P., Jones, P. W. \& Wallis, T. S. (2000). Salmonella enterica serovar typhimurium and dublin can lyse macrophages by a mechanism distinct from apoptosis. Infect Immun 68, 3744-3747.
Wilcock, B. P. \& Schwartz, K. J. (1992). Salmonellosis. In Diseases of Swine, 7th edn, pp. 570-583. Edited by A. D. Leman and others. Ames, IA: Iowa State University Press.

Williams Smith, H. \& Halls, S. (1966). The immunity produced by a rough $S$. dublin variant against Salmonella typhimurium and Salmonella choleraesuis infection in guinea-pigs. J Hyg 64, 357-359.

Williams Smith, H. \& Halls, S. (1968). The simultaneous oral administration of Salmonella dublin, S. typhimurium and $S$. choleraesuis to calves and other animals. J Med Microbiol 1, 203-209.

Winkler, G. C. (1988). Pulmonary intravascular macrophages in domestic animal species: review of structural and functional properties. Am J Anat 181, 217-234.

Zychlinsky, A., Prevost, M. C. \& Sansonetti, P. J. (1992). Shigella flexneri induces apoptosis in infected macrophages. Nature 358, $167-169$.

Received 10 January 2000; revised 23 March 2000; accepted 27 March 2000. 